\title{
Colistin: recent data on pharmacodynamics properties and clinical efficacy in critically ill patients
}

Argyris S Michalopoulos ${ }^{1,2}$ and Matthew E Falagas $2,3,4^{*}$

\begin{abstract}
Recent clinical studies performed in a large number of patients showed that colistin "forgotten" for several decades revived for the management of infections due to multidrug-resistant (MDR) Gram-negative bacteria (GNB) and had acceptable effectiveness and considerably less toxicity than that reported in older publications. Colistin is a rapidly bactericidal antimicrobial agent that possesses a significant postantibiotic effect against MDR Gram-negative pathogens, such as Pseudomonas aeruginosa, Acinetobacter baumannii, and Klebsiella pneumoniae. The optimal colistin dosing regimen against MDR GNB is still unknown in the intensive care unit (ICU) setting. A better understanding of the pharmacokinetic-pharmacodynamic relationship of colistin is urgently needed to determine the optimal dosing regimen. Although pharmacokinetic and pharmacodynamic data in ICU patients are scarce, recent evidence shows that the pharmacokinetics/pharmacodynamics of colistimethate sodium and colistin in critically ill patients differ from those previously found in other groups, such as cystic fibrosis patients. The AUC:MIC ratio has been found to be the parameter best associated with colistin efficacy. To maximize the AUC:MIC ratio, higher doses of colistimethate sodium and alterations in the dosing intervals may be warranted in the ICU setting. In addition, the development of colistin resistance has been linked to inadequate colistin dosing. This enforces the importance of colistin dose optimization in critically ill patients. Although higher colistin doses seem to be beneficial, the lack of colistin pharmacokinetic-pharmacodynamic data results in difficulty for the optimization of daily colistin dose. In conclusion, although colistin seems to be a very reliable alternative for the management of life-threatening nosocomial infections due to MDR GNB, it should be emphasized that there is a lack of guidelines regarding the ideal management of these infections and the appropriate colistin doses in critically ill patients with and without multiple organ failure.
\end{abstract}

\section{Colistin's pharmacodynamic properties}

Colistimethate sodium (CMS) is an inactive prodrug of colistin that exhibits a low level of protein binding. It is not stable in vitro and in vivo and is hydrolyzed in human plasma, creating a complex mixture of partially sulphomethylated derivatives with the potential to produce up to 32 different products, including colistin [1]. After administration of CMS, colistin appears in plasma rapidly. Colistin is approximately $50 \%$ bound to human plasma. Peak serum levels after intravenous (i.v.) administration are achieved within $10 \mathrm{~min}$. They appeared

\footnotetext{
* Correspondence: m.falagas@aibs.gr

${ }^{2}$ Alfa Institute of Biomedical Sciences (AIBS), 9 Neapoleos Street, 15123 Marousi, Greece

Full list of author information is available at the end of the article
}

higher but declined more rapidly than those achieved after i.m. administration [2].

Colistin (base) is more active than CMS. Serum halflife of CMS is approximately 1.5-2 hours (h) after i.v. administration and 2.75 to $3 \mathrm{~h}$ after i.m. administration in healthy subjects, whereas serum half-life for CMS administered i.v. is more than $4 \mathrm{~h}$. Old reports have suggested that colistin is poorly distributed to the pleural cavity, lung parenchyma, bones, and cerebrospinal fluid (CSF) (15\% to $25 \%)$.

CMS is eliminated predominantly by the kidneys. It should be noted that after CMS i.v. administration, approximately $60 \%$ of CMS is excreted unchanged in the urine via glomerular filtration during the first $24 \mathrm{~h}$. In renal failure, the renal excretion of CMS is decreased resulting in a higher conversion to colistin and

\section{SpringerOpen $^{\circ}$}


prolongation of half-life [3]. On the contrary, colistin is eliminated predominantly by the nonrenal route by means of mechanisms not yet fully understood [4]. However, in humans, colistin is not absorbed from the gastrointestinal tract and no biliary excretion has been reported.

The pharmacodynamic (PD) properties of colistin, such as minimal inhibitory concentration (MIC), mutation prevention concentration, population analysis profile, bacterial-killing kinetics, and the postantibiotic effect (PAE) against multidrug-resistant (MDR) Gramnegative bacteria (GNB), such as Pseudomonas aeruginosa, Acinetobacter baumannii, and Klebsiella pneumoniae, have been examined in recent studies [5,6]. Based on the study by Owen et al. [5] colistin seems to be very active in the initial killing of $A$. baumannii, even with $0.5 \times$ MIC, exhibiting a concentration-dependent bacterial-killing mechanism. Modest positive PAEs of colistin were observed at relatively high concentrations $(\geq 16 \times$ MIC), which are not achieved in clinical practice. The most significant finding of the study was the substantial regrowth occurring at $24 \mathrm{~h}$ even at colistin concentrations up to $64 \times$ MIC and the minor or negative PAE of colistin [7]. These findings were consistent with the hetero-resistance of A. baumannii isolates to colistin, reported in previous studies, suggesting that CMS monotherapy and extended-interval dosage regimens may be problematic for the effective treatment of nosocomial infections caused by colistin-heteroresistant $A$. baumannii in the intensive care unit (ICU) setting [7].

Poudyal et al. [6] found initial rapid killing against $K$. pneumoniae strains even at the lowest colistin concentration. Similarly with the previous study dealing with the colistin pharmacodynamics against MDR A. baumannii, colistin exhibited no PAE at up to $64 \times$ MIC, regrowth in the majority of isolates at $4 \mathrm{~h}$ and heteroresistance to colistin in MDR but colistin-susceptible $K$. pneumoniae strains. These findings suggest that CMS monotherapy and extended-interval dosage regimens, as has been aforementioned for $A$. baumannii isolates, may promote colistin resistance in MDR K. pneumoniae strains

\section{Colistin's pharmacokinetic properties}

The main pharmacokinetics (PK) of colistin are presented in Table 1. Few studies have addressed the PKs of CMS and colistin in humans, especially in the ICU setting. It should be emphasized that significant pharmacodynamic parameters, such as Cmax/MIC ratio, AUC/MIC, and T > MIC that could best predict colistin efficacy and safety have not yet been clearly defined in humans in critically ill patients. For this reason, the optimum target for colistin Cmax/MIC ratio is not yet known. In addition, there is still a lack of $\mathrm{PK} / \mathrm{PD}$ information to optimize colistin doses in humans, especially those who are hospitalized in the ICUs. A better understanding of the CMS and colistin (base) PKs could be beneficial for colistin use in humans. It is known that CMS and colistin (base) PKs differ, given that they have different structures, antibacterial activity, and toxicity.

Bergen et al. [8] examined the pharmacokinetics of colistin in an in vitro pharmacokinetic/pharmacodynamic model. Three intermittent dosage regimens involving 8-h, 12-h, and 24-h dosage intervals (Cmax of 3.0, 4.5 , or $9.0 \mathrm{mg} / \mathrm{L}$, respectively) were administered in humans. Antibacterial activity and emergence of resistance were investigated during the 72-hour treatment period using two strains of $P$. aeruginosa. No difference in overall bacterial killing was found. However, the 8hourly regimens appeared most effective at minimizing the onset of resistance. This study additionally showed that the AUC:MIC ratio of total and unbound colistin is the index that best predicts the antibacterial activity against $P$. aeruginosa, superior to Cmax/MIC, suggesting that time-averaged exposure to colistin is more important than the achievement of high peak concentrations. The PK/PD relationship of colistin against $P$. aeruginosa has been examined recently in a vitro model. A significant finding of the study was that colistin efficacy against $P$. aeruginosa was best correlated with the AUC: MIC ratio of total and unbound colistin rather than the $\mathrm{Cmax} / \mathrm{MIC}$ ratio. As a consequence, the time-averaged exposure to colistin is a more important target in the clinical practice than the achievement of high colistin peak concentrations [9].

Steady-state pharmacokinetics of colistin has been recently examined in 13 adult patients with ventilatorassociated pneumonia (VAP) caused by GNB. Patients were treated with CMS: 2 million (m.) units that are equivalent with $174 \mathrm{mg}$ CMS, administered i.v. every 8 hours, for at least 2 days. Patients received a mean of $2.19 \mathrm{mg} / \mathrm{kg}$ of CMS per dose. At steady-state, apparent volume of distribution $(\mathrm{Vd} / \mathrm{fm})$ was $1.5 \pm 1.1 \mathrm{~L} / \mathrm{kg}$. $\mathrm{Cmax} / \mathrm{MIC}$ ratio and $\mathrm{AUC0}-24 / \mathrm{MIC}$ ratio (for $\mathrm{MIC}=2$ $\mathrm{mcg} / \mathrm{ml}$ ) were $1.1 \pm 0.5$ and $17.3 \pm 9.3$, respectively. The authors also examined the colistin concentration in BAL, which was found to be undetectable. Based on these findings, it seems that the reported daily colistin dose of $6 \mathrm{~m}$. units $(2 \mathrm{~m}$. units administered every 8 hours) resulted in suboptimal serum colistin concentrations and undetectable colistin concentrations in BAL in critically ill patients [10].

It should be noted that recently Dudhani et al. used two murine infection models to identify the most predictive PK/PD index of the antibacterial activity of colistin against $P$. aeruginosa and $A$. baumannii strains. The authors reported that fAUC/MIC was the most predictive $\mathrm{PK} / \mathrm{PD}$ index that correlated best with colistin 
efficacy against these Gram-negative pathogens in both thigh and lung infection models. These studies highlighted the importance of achieving adequate time-averaged exposure to colistin across the day $[11,12]$. These studies performed in animals will facilitate efforts to define in the near future the more rational design of CMS doses in humans, especially in the ICU setting.

\section{Optimizing colistin dose based on PK/PD properties}

The optimal dose of colistin has not been determined in the ICU setting, because since there is a lack of relative clinical studies. In addition, there is lack of colistin's PK/PD data in critically ill patients. Reliable colistin PK/ PD data, a better understanding of these data, and recent randomized, controlled trials are necessary to redefine appropriate colistin doses. This strategy relates to all potential routes of colistin administration to maximize colistin clinical efficacy associated with minimal adverse effects. In addition, there is discrepancy regarding the recommended doses of colistin (CMS) worldwide. This fact is mainly based on two major parameters: 1) the amount of colistin included in each vial of colistin in different countries is different; and 2) some vials refer to CMS but others in colistin base. It seems that the best way to avoid confusion related to colistin dosing is to base the doses on international units. Pure colistin base has been assigned a potency of $30,000 \mathrm{IU}$ per $\mathrm{mg}$, and CMS has a potency of $12,500 \mathrm{IU}$ per mg. Thus, recommendations regarding dosing of colistin should clearly refer to colistin base or colistimethate sodium to avoid possible confusion. The recommended doses of CMS in patients with normal renal function, those with renal failure, and those who undergo renal replacement therapy or peritoneal dialysis are presented in Table 2.

The steady-state colistin serum concentrations have been measured in 14 septic patients with stable renal function in a general ICU after i.v. administration of CMS. The CMS dose was $225 \mathrm{mg}$ administered every 8 or $12 \mathrm{~h}$ for at least 2 days. At steady state, mean maximum and minimum colistin concentrations were 2.93 and $1.03 \mathrm{mg} / \mathrm{L}$, respectively, whereas mean apparent total body clearance was $13.6 \mathrm{~L} / \mathrm{h}$, apparent volume of distribution was $139.9 \mathrm{~L}$, and $\mathrm{t}(1 / 2)$ was $7.4 \mathrm{~h}$. Cmax/ MIC ratio displayed a wide range of values. The authors reported that with colistin sensitivity defined as a MIC break point $\leq 2 \mu \mathrm{g} / \mathrm{mL}$, the Cmax levels achieved with this colistin dose would most probably lead to suboptimal Cmax/MIC ratios for many isolated strains in the upper range of these MIC values. The authors concluded that higher doses of CMS should be considered in critically ill patients [13].

CMS and colistin PKs have been recently examined in 18 adult critically ill patients who received i.v. colistin for infections caused by MDR-GNB. CMS was administered at a dose of $3 \mathrm{~m}$. units $(240 \mathrm{mg}$ ) every $8 \mathrm{~h}$ (or 160 mg every $8 \mathrm{~h}$ if creatinine clearance was $<50 \mathrm{ml} / \mathrm{min}$ ). The clearance of CMS was $13.7 \mathrm{~L} / \mathrm{h}$. For colistin, the estimated half-life was $14.4 \mathrm{~h}$. The predicted maximum concentrations of drug in plasma were $0.60 \mathrm{mg} / \mathrm{L}$ for the first dose and $2.3 \mathrm{mg} / \mathrm{L}$ at steady state. After the first few doses, colistin concentrations were below the Clinical and Laboratory Standards Institute MIC breakpoint of $2 \mathrm{mg} / \mathrm{L}$ for $P$. aeruginosa and Enterobacteriaceae. In addition, at steady state, plasma concentrations were below the MIC breakpoints for many of the cases. At daily colistin doses of $9 \mathrm{~m}$. units $(3 \mathrm{~m}$. units administered every $8 \mathrm{~h}$ ), it would take 2 -3 days before the steady-state concentration was achieved. A significant finding of the study was that colistin displayed a significantly longer half-life in relation to the dosing interval. The authors speculated that a loading colistin dose of 9 or $12 \mathrm{~m}$. units along with a maintenance dose of $4.5 \mathrm{~m}$. units administered every $12 \mathrm{~h}$ is necessary in critically ill patients [14].

Another important aspect to be determined is the colistin frequency of dosing in critically ill patients. The PKs of three different CMS daily doses (3 m. units every $8 \mathrm{~h} 4.5 \mathrm{~m}$. units every $12 \mathrm{~h}$ and $9 \mathrm{~m}$. units every $24 \mathrm{~h}$ ) have been recently examined by Daikos et al. [15]. The authors evaluated the bactericidal activity of serum containing various concentrations of colistin against $P$. aeruginosa with a MIC $1 \mu \mathrm{g} / \mathrm{ml}$. Mean serum C (max) of 


\section{Table 2 Recommended doses of i.v. colistin (CMS) in critically ill patients}

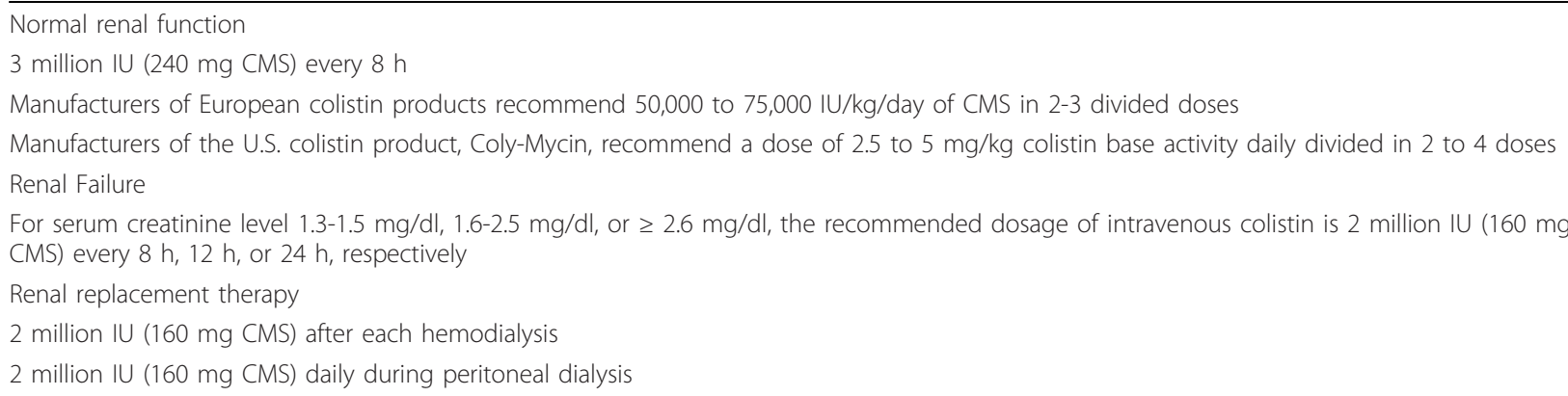

colistin were $3.34,2.98$, and $5.63 \mu \mathrm{g} / \mathrm{ml}$, respectively. All serum samples containing colistin $>4 \mu \mathrm{g} / \mathrm{ml}$ (serum colistin concentration $/ \mathrm{MIC}>4$ ) eliminated $P$. aeruginosa, whereas only $40 \%$ of samples containing colistin < $4 \mu \mathrm{g} / \mathrm{ml}$ resulted in complete bacterial killing. Based on these findings, the currently used colistin dosing regimens might not provide the most effective therapy and therefore might justify administering larger colistin doses in longer intervals. However, although the potential for a longer dosing interval may be an option in critically ill patients, some studies found that when the intervals of colistin doses increase, the prevalence of colistin resistance also increases.

Clearance of CMS and colistin was found to be lower, whereas conversion of CMS to colistin and overall colistin exposure were increased in patients with renal failure compared with healthy subjects. No clinical data exist on colistin dosing for patients receiving continuous renal replacement therapy. Based on the PK properties of colistin, the recommended dose of colistin in this group of patients is $2.5 \mathrm{mg} / \mathrm{kg}$ q 48 h. However, there are serious doubts about this recommendation. It is likely that higher colistin dosage (e.g., 2 to $3 \mathrm{mg} / \mathrm{kg}$ every $12 \mathrm{~h}$ ) is necessary. In a patient undergoing continuous venovenous hemodiafiltration, conversion of CMS to colistin was rapid, and the terminal half-lives of CMS and colistin were 6.8 and $7.5 \mathrm{~h}$, respectively [16]. Based on older studies, in patients with renal failure undergoing peritoneal dialysis, approximately $1 \mathrm{mg} / \mathrm{h}$ of colistin is removed from the patient and approximately $16 \%$ of the total colistin dose is removed during a 2 -h peritoneal dialysis session. Because of this poor clearance, the recommended dose of colistin should be $2 \mathrm{mg} / \mathrm{kg} /$ day.

During the past decade, inhaled colistin has been used for the treatment of nosocomial pneumonia or VAP due to MDR GNB, mostly $P$. aeruginosa and A. baumannii, to improve lung parenchyma penetration. Although administration of colistin via inhalation has been adopted and recommended to improve lung parenchyma penetration in the adjunct treatment of MDR pneumonia or VAP, there are until now few data on the PKs of colistin after inhalation. In addition, no study has been performed to assess the colistin concentrations achieved in the pulmonary epithelial lining fluid, which is the target site for antibiotics, in the treatment of pneumonia. The first study that evaluated the colistin pharmacokinetics postinhalation was conducted by Ratjen et al. [17] in patients with cystic fibrosis. In this multicenter study, a single dose of CMS ( $2 \mathrm{~m}$. units) was administered via inhalation to assess sputum, serum, and urine concentrations. An interesting finding of this study was that the maximum sputum concentrations of colistin were at least 10 times higher than those proposed by the British Society for Antimicrobial Chemotherapy. Although sputum drug concentrations decreased after a peak at $1 \mathrm{~h}$, the mean colistin concentrations remained above $4 \mathrm{mg} / \mathrm{L}$ after $12 \mathrm{~h}$. Serum concentrations of colistin reached their maximum at $1.5 \mathrm{~h}$ after inhalation and decreased thereafter. A mean of 4.3 $\pm 1.3 \%$ of the inhaled dose was detected in urine.

Lu et al. [18] compared lung tissue deposition and antibacterial efficiency between nebulized and intravenous administration of colistin in piglets with pneumonia caused by $P$. aeruginosa. CMS was administered either by nebulization every $12 \mathrm{~h}$ or i.v. every $8 \mathrm{~h}$. The fraction of CMS reaching the respiratory tract was $60 \%$ of the initial dose. An interesting finding of this study was that colistin was undetected in lung tissue after intravenous infusion. On the contrary, median colistin peak lung concentration after nebulization was $2.8 \mu \mathrm{g} / \mathrm{g}$. After three consecutive CMS aerosols, peak tissue concentrations were found higher than MIC, indicating significant distal lung deposition. In the aerosol group of piglets, peak lung tissue concentrations were significantly higher in lung segments with mild pneumonia (median $=10.0 \mu \mathrm{g} / \mathrm{g})$ compared with lung segments with severe pneumonia (median $=1.2 \mu \mathrm{g} / \mathrm{g} ; p<0.01$ ). After $24 \mathrm{~h}$ of colistin treatment, $67 \%$ of pulmonary segments had bacterial counts $<10^{2} \mathrm{cfu} / \mathrm{g}$ after nebulization and $28 \%$ after i.v. administration $(p<0.001)$. On the contrary, in control animals, $12 \%$ of lung segments 
had bacterial counts $<10^{2} \mathrm{cfu} / \mathrm{g} 49 \mathrm{~h}$ after bronchial inoculation.

Although these data seem promising, it is not known whether they can be extrapolated to critically ill patients with MDR nosocomial pneumonia in the ICU setting, who may display different pharmacokinetics parameters compared with patients with cystic fibrosis. Hence, pharmacokinetic data regarding inhaled colistin in ICU patients with MDR VAP are very much warranted.

Only a few case reports in the literature deal with the intrathecal administration of colistin for the treatment of ventriculitis and shunt infections due to carbapenem resistant $P$. aeruginosa and A. baumannii [19-21]. Markantonis et al. [22] examined recently colistin concentrations in serum and CSF samples in five critically ill patients who received CMS for CNS infections due to MDR GNB. The objective of this study was to investigate colistin's penetration into the CSF. However, they found low penetration level (5\%) suggesting inadequate bactericidal colistin concentrations in the CSF.

The treatment of postneurosurgical meningitis or ventriculitis or CNS shunt infection due to MDR GNB, such as $A$. baumannii and $P$. aeruginosa, is a difficult clinical problem and is associated with high mortality rates mainly due to the limited penetration of colistin into the CSF. There are few case reports dealing with the successful management of postneurosurgical ventriculitis due to MDR A. baumannii or $P$. aeruginosa strains treated successfully and safely with CMS administered by the intrathecal or intraventricular route. The dosage of colistin (base) for intraventricular administration ranges from $5 \mathrm{mg}$ to $20 \mathrm{mg}$ per day (divided in 1 or 2 doses). In our patients, we administer 500,000 IU CMS once per day intraventricularly or directly into CSF for 2 consecutive days followed by 300,000 IU once per day for the following 57 days. The median time necessary to obtain CSF sterilization seems to be approximately 5 days. Toxicity probably or possibly related to the topical administration of colistin is noted in approximately $15 \%$ of patients.

\section{Colistin's clinical efficacy in critically ill patients}

In a recently published study, 258 adult critically ill patients (mean age 61 years) received i.v. colistin for at least 72 hours for microbiologically documented MDR Gram-negative infections mainly due to $A$. baumannii (65.9\%) and P. aeruginosa (26.4\%). The mean duration of hospital and ICU stays until the start of colistin administration for the index infection was 18.3 and 11.4 days, respectively. The mean duration of colistin administration was 17.9 days and the interquartile range was 10-22 days. Cure of infection occurred in $79.1 \%$ of patients. An interesting finding of this study was that nephrotoxicity occurred in only $10 \%$ of patients [23]. Similar rates of nephrotoxicity are reported by other studies [24-26]. please, delete reference No 11 On the contrary, Koomanachai et al. and Kim et al. reported a colistin-induced nephrotoxicity in approximately $30 \%$ of patients $[27,28]$.

Apart from adults, intravenous colistin also has been administered with safety and efficacy in children and neonates, including preterm and extremely low birth weight neonates [29-31].

\section{Conclusions}

Numerous recent clinical studies have confirmed that colistin is an efficient antimicrobial agent against nosocomial infections, including bacteremia, ventilator-associated pneumonia, urinary tract infection, and meningitis due to MDR GNB, such as $P$. aeruginosa, $A$. baumannii, and $K$. pneumonia, with an acceptable safety profile. Whereas colistin is mainly administered i.v. in critically ill patients, it can be safely be administered by inhalation in patients with pneumonia/VAP or intrathecally in patients with meningitis due to MDR GNB. Although colistin PK/PD data are scarce in ICU patients, recent evidence shows that the PK/PD properties of CMS and colistin are different in critically ill patients compared with other groups, such as patients with cystic fibrosis. A better understanding of colistin PK-PD properties is urgently needed to determine the optimal dosing regimen in colistin monotherapy or combination therapy for the effective management of life-threatening nosocomial infections due to MDR GNB in critically ill patients.

\section{Author details}

${ }^{1}$ Department of Critical Care Medicine, Henry Dunant Hospital, Mesogeion 107, 11526 Athens, Greece ${ }^{2}$ Alfa Institute of Biomedical Sciences (AIBS), 9 Neapoleos Street, 15123 Marousi, Greece ${ }^{3}$ Department of Medicine, Henry Dunant Hospital, Mesogeion 107, 11526 Athens, Greece ${ }^{4}$ Department of Medicine, Tufts University School of Medicine, Boston, MA, USA

\section{Authors' contributions}

MA wrote the first draft of the manuscript. MEF did substantial revisions. Both authors approved the final version of the manuscript.

\section{Competing interests}

Argyris Michalopoulos declares that he has no competing interests. Mathew E. Falagas has participated in advisory boards of Pfizer, Astellas, and Bayer and has received lecture honoraria from Merck, Pfizer, AstraZeneca, Astellas, Cipla, Novartis, and Glenmark.

Received: 19 April 2011 Accepted: 2 August 2011

Published: 2 August 2011

\section{References}

1. Li J, Nation RL, Milne RW, Turnidge JD, Coulthard K: Evaluation of colistin as an agent against multi-resistant Gram-negative bacteria. Int J Antimicrob Agents 2005, 25:11-25.

2. Froman J, Gross L, Curatola S: Serum and urine levels following parenteral administration of sodium colistimethate to normal individuals. J Urol 1970, 103:210-214. 
3. Gupta S, Govil D, Kakar PN, Prakash O, Arora D, Das S, Govil P, Malhotra A: Colistin and polymyxin B: a re-emergence. Indian J Crit Care Med 2009, 13:49-53.

4. Li J, Nation RL, Turnidge JD, Milne RW, Coulthard K, Rayner CR, Paterson DL: Colistin: the re-emerging antibiotic for multidrug-resistant Gramnegative bacterial infections. Lancet Infect Dis 2006, 6:589-601.

5. Owen RJ, Li J, Nation RL, Spelman D: In vitro pharmaco-dynamics of colistin against Acinetobacter baumannii clinical isolates. J Antimicrob Chemother 2007, 59:473-477.

6. Poudyal A, Howden BP, Bell JM, Gao W, Owen RJ, Turnidge JD, Nation RL, $\mathrm{Li} \mathrm{J}$ : In vitro pharmacodynamics of colistin against multidrug-resistant Klebsiella pneumoniae. J Antimicrob Chemother 2008, 62:1311-1318.

7. Li J, Rayner CR, Nation RL, Owen RJ, Spelman D, Tan KE, Liolios L: Heteroresistance to colistin in multidrug-resistant Acinetobacter baumannii. Antimicrob Agents Chemother 2006, 50:2946-2950.

8. Bergen PJ, Li J, Nation RL, Turnidge JD, Coulthard K, Milne RW: Comparison of once-, twice- and thrice daily dosing of colistin on antibacterial effect and emergence of resistance: studies with Pseudomonas aeruginosa in an in vitro pharmacodynamic model. J Antimicrob Chemother 2008, 61:636-642.

9. Bergen PJ, Bulitta JB, Forrest A, Tsuji BT, Li J, Nation RL: Pharmacokinetic (PK)/Pharmacodynamic (PD) investigation of colistin (C) against Pseudomonas aeruginosa (Pa) using an in vitro model. Antimicrob Agents Chemother 2010, 54:3783-3789.

10. Imberti R, Cusato M, Villani P, Carnevale L, lotti GA, Langer M, Regazzi M: Steady-state pharmacokinetics and bronchoalveolar lavage concentration of colistin in critically-ill patients after intravenous colistin methanesulphonate administration. Chest 2010, 138:1333-1339.

11. Dudhani RV, Turnidge JD, Coulthard K, Milne RW, Rayner CR, Li J, Nation RL: Elucidation of the pharmacokinetic/pharmacodynamic determinant of colistin activity against Pseudomonas aeruginosa in murine thigh and lung infection models. Antimicrob Agents Chemother 2010, 54:1117-1124.

12. Dudhani RV, Turnidge JD, Nation RL, Li J: fAUC/MIC is the most predictive pharmacokinetic/pharmacodynamic index of colistin against Acinetobacter baumannii in murine thigh and lung infection models. $J$ Antimicrob Chemother 2010, 65:1984-1990.

13. Markou N, Markantonis SL, Dimitrakis E, Panidis D, Boutzouka E, Karatzas S, Rafailidis P, Apostolakos H, Baltopoulos G: Colistin serum concentrations after intravenous administration in critically ill patients with serious multidrug-resistant, gram-negative bacilli infections: a prospective, open-label, uncontrolled study. Clin Ther 2008, 30:143-151.

14. Plachouras D, Karvanen M, Friberg LE, Papadomichelakis E, Antoniadou A, Tsangaris I, Karaiskos I, Poulakou G, Kontopidou F, Armaganidis A, Cars O, Giamarellou H: Population pharmacokinetic analysis of colistin methanesulfonate and colistin after intravenous administration in critically-ill patients with infections caused by gram-negative bacteria. Antimicrob Agents Chemother 2009, 53:3430-3436.

15. Daikos GL, Skiada A, Pavleas J, Vafiadi C, Salatas K, Tofas P, Tzanetou K, Markogiannakis A, Thomopoulos G, Vafiadi I, Petrikkos G: Serum bactericidal activity of three different dosing regimens of colistin with implications for optimum clinical use. J Chemother 2010, 22:175-178.

16. Li J, Rayner CR, Nation RL, Deans R, Boots R, Widdecombe N, Douglas A Lipman J: Pharmacokinetics of colistin methanesulfonate and colistin in a critically ill patient receiving continuous venovenous hemodiafiltration Antimicrob Agents Chemother 2005, 49:4814-4815

17. Ratjen F, Rietschel E, Kasel D, Schwiertz R, Starke K, Beier H, van Koningsbruggen S, Grasemann H: Pharmacokinetics of inhaled colistin in patients with cystic fibrosis. J Antimicrob Chemother 2006, 57:306-311.

18. Lu Q, Girardi C, Zhang M, Bouhemad B, Louchahi K, Petitjean O, Wallet F, Becquemin $\mathrm{MH}$, Le Naour G, Marquette $\mathrm{CH}$, Rouby JJ: Nebulized and intravenous colistin in experimental pneumonia caused by Pseudomonas aeruginosa. Intensive Care Med 2010, 6:1147-1155.

19. Fernández-Viladrich $\mathrm{P}$, Corbella X, Corral L, Tubau F, Mateu A: Successfu treatment of ventriculitis due to carbapenem-resistant Acinetobacter baumannii with intraventricular colistin sulfomethate sodium. Clin Infect Dis 1999, 28:916-917.

20. Gump WC, Walsh JW: Intrathecal colistin for treatment of highly resistant Pseudomonas ventriculitis: case report and review of the literature. $J$ Neurosurg 2005, 102:915-917.
21. Quinn AL, Parada JP, Belmares J, O'Keefe JP: Intrathecal colistin and sterilization of resistant Pseudomonas aeruginosa shunt infection. Ann Pharmacother 2005, 39:949-952.

22. Markantonis SL, Markou N, Fousteri M, Sakellaridis N, Karatzas S, Alamanos I, Dimopoulou E, Baltopoulos G: Penetration of colistin into cerebrospinal fluid. Antimicrob Agents Chemother 2009, 53:4907-4910.

23. Falagas $M$, Rafailidis $P$, loannidou $E$, Alexiou V, Matthaiou $D$, Karageorgopoulos D, Kapaskelis A, Nikita D, Michalopoulos A: Colistin therapy for microbiologically documented multidrug-resistant Gram (-) bacterial infections: a retrospective cohort study of 258 patients. Int J Antimicrob Agents 2010, 35:194-199.

24. Falagas ME, Fragoulis KN, Kasiakou SK, Sermaidis GJ, Michalopoulos A: Nephrotoxicity of intravenous colistin: a prospective evaluation. Int J Antimicrob Agents 2007, 26:504-507.

25. Santamaría C, Mykietiuk A, Temporiti E, Stryjewski ME, Herrera F, Bonvehi P: Nephrotoxicity associated with the use of intravenous colistin. Scand J Infect Dis 2009, 41:767-769.

26. Cheng CY, Sheng WH, Wang JT, Chen YC, Chang SC: Safety and efficacy of intravenous colistin (colistin methanesulphonate) for severe multidrugresistant Gram-negative bacterial infections. Int J Antimicrob Agents 2010, 35:297-300.

27. Koomanachai $P$, Tiengrim S, Kiratisin P, Thamlikitkul V: Efficacy and safety of colistin (colistimethate sodium) for therapy of infections caused by multidrug-resistant Pseudomonas aeruginosa and Acinetobacter baumannii in Siriraj Hospital, Bangkok, Thailand. Int J Infect Dis 2007, 11:402-406.

28. Kim J, Lee $\mathrm{KH}$, Yoo S, Pai H: Clinical characteristics and risk factors of colistin-induced nephrotoxicity. Int J Antimicrob Agents 2009, 34:434-438.

29. Iosifidis E, Antachopoulos C, loannidou M, Mitroudi M, Sdougka M, DrossouAgakidou V, Tsivitanidou M, Roilides E: Colistin administration to pediatric and neonatal patients. Eur J Pediatr 2010, 169:867-874.

30. Celebi S, Hacimustafaoglu M, Koksal N, Ozkan H, Cetinkaya M: Colistimethate sodium therapy for multidrug-resistant isolates in pediatric patients. Pediatr Int 2010, 52:410-414.

31. Jajoo M, Kumar V, Jain M, Kumari S, Manchanda V: Intravenous colistin administration in neonates. Pediatr Infect Dis J 2011, 30:218-221.

doi:10.1186/2110-5820-1-30

Cite this article as: Michalopoulos and Falagas: Colistin: recent data on pharmacodynamics properties and clinical efficacy in critically ill patients. Annals of Intensive Care 2011 1:30.

\section{Submit your manuscript to a SpringerOpen ${ }^{\circ}$ journal and benefit from:}

- Convenient online submission

- Rigorous peer review

- Immediate publication on acceptance

- Open access: articles freely available online

- High visibility within the field

- Retaining the copyright to your article

Submit your next manuscript at $>$ springeropen.com 